\title{
Collimation Experience at RHIC $^{*}$
}

\author{
K.A. Drees, R.Fliller; D. Trbojevic * and V. Kain ${ }^{\dagger}$ \\ "Brookhaven National Laboratory, Upton, NY 11973 :. \\ †CERN; Geneva, Switzerland.
}

\begin{abstract}
In the Relativistic Heavy. Ion Collider (RHIC) the abort kicker magnets are the limiting aperture. Continuous losses : at this location could deteriorate the kicker performance. In addition, losses especially in the triplet area cause backgrounds in :the experimental detectors. The RHIC one-stage collimation system was used to reduce these backgrounds as well as losses at the abort kickers. Collimation performance and results from various runs with even and uneven species ( $\mathrm{Au}-\mathrm{Au}, \mathrm{pp}$ and $\mathrm{d}-\mathrm{Au}$ ) are presented and compared. Upgrades of the system for the upcoming high luminosity runs are outlined.:
\end{abstract}

\section{INTRODUCTION}

Beam halo, large beam profiles and beam losses induce high experimental backgrounds throughout the stores as well as contribute to the reduction of the lifetime of accelerator components. In superconducting machines quenches due to uncontrolled beam losses during beam steering, the acceleration ramp or fault conditions are likely. Collimators used as the limiting aperture can help prevent damage. Figure 1 -sketches the geometry of RHIC : with the collimators and the five RHIC experiments.

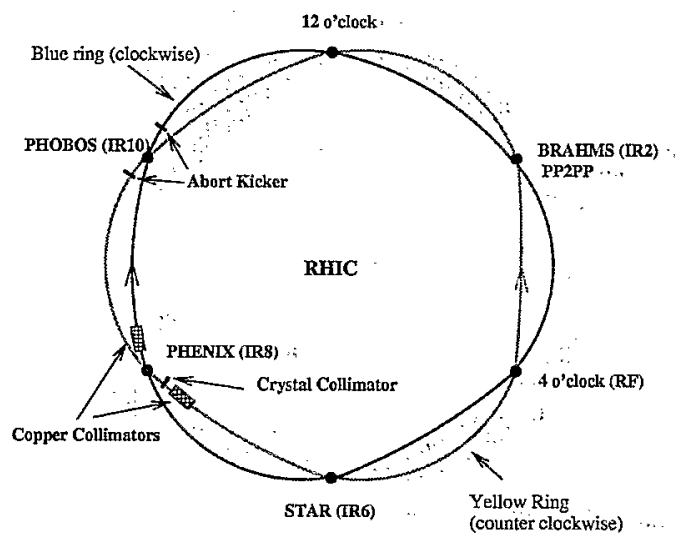

FIGURE 1. Location of the collimators and experiments in the RHIC rings.

Table 1 lists : all RHIC runs to date. The operation period in the year 2001/2002 consisted of a heavy ion run followed by 8 weeks : of a polarized proton .run: Both runs had different needs for collimation. During the Au-run several ramps were introduced implementing $\beta^{*}$-squeezes from $10 \mathrm{~m}$ to $5 \mathrm{~m}$, from $10 \mathrm{~m}$ to $2 \mathrm{~m}$ and fi-
TABLE 1. L List of RHIC runs 1999-2003.

\begin{tabular}{|c|c|c|c|}
\hline year & run & Energy & $\beta^{*}$ \\
\hline 1999 & pilot au & $10 \mathrm{GeV}$ & $10 \mathrm{~m}$ all \\
\hline 2000 & rhic_au_00 & $70 \mathrm{GeV}$ & $3 \mathrm{~m}, 8 \mathrm{~m}$ \\
\hline 2001 & rhic_au_01 & $100 \mathrm{GeV}$ & $2 \mathrm{~m} ; 1 \mathrm{~m}$ \\
\hline 2002 & rhic_pp_02 & $100 \mathrm{GeV}$ & $3 \mathrm{~m}$ all \\
\hline \multirow[t]{2}{*}{2003} & rhic_dau_03 & $: 100 \mathrm{GeV}$ & $2 \mathrm{~m}, 3 \mathrm{~m}, 4 \mathrm{~m}$ \\
\hline & rhic_pp_03 & $100 \mathrm{GeV}$ & $1 \mathrm{~m}, 2 \mathrm{~m}, 10 \mathrm{~m}$ \\
\hline
\end{tabular}

nally from $10 \mathrm{~m}$ to $1 \mathrm{~m}$ (at PHENIX only). There were no squeezes during the proton run, instead $\beta^{*}=3 \mathrm{~m}$ was used for injection as well as storage for all IRs. In the year 2003, the pp run was preceded by a run with uneven species (d and $A u)$. In both runs $\beta^{*}$. was squeezed to several different values at the IRs. The many configurations introduced an increased demand for collimation in the FY03 runs.

\section{LAYOUT'}

The RHIC : collimation system [1] layout is shown in ... figure 2.

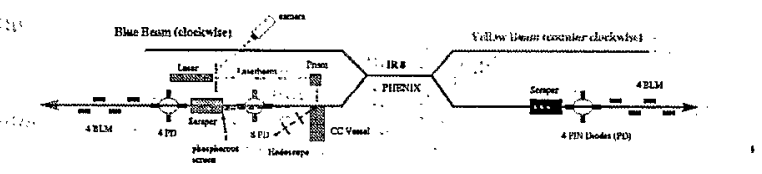

FIGURE 2. The RHIC collimation system

It consists of two $450 \mathrm{~mm}$ long L-shaped copper scrapers placed downstream of the PHENIX detector in each ring.. Each collimator is moved by three stepper motors, which control the hörizontal and vertical positions and 
rotate the collimator about the vertical axis. The step size is approximately $0.5 \mu \mathrm{m}$. Fully retracted the vertical jaws are about $56 \mathrm{~mm}$ and the horizontal jaws about $52 \mathrm{~mm}$ from the center. of the beam:pipe. In addition the yellow (counter-clockwise) ring has a $5 \mathrm{~mm}$ long, O-shaped silicon crystal. The crystal is bent $0.44 \mathrm{mrad}$. More details can be found in [1]. Because of the negligible dispersion at the location of the collimators [2], the scrapers cannot be used for momentum collimation. Since RHIC: so far lacks any other collimators; a combination of fast kickers and the scrapers was used to excite and remove offmomentum beam particles during the last run [3]. A dedicated gap cleaning system including additional kickers is currently being installed during the FY03 shutdown.

\section{HEAVY:ION OPERATION:}

During the 2001 au_01 and the later dau_03 run the ions were accelerated up to $\gamma \approx 107$. During the acceleration ramp various processes, such as orbit variations and radial shifts, are potentially leading to beam losses. It turned out that the abort system kickers acted as limiting aperture (see [2] for more details). In the run au_01 the collimators were moved in to a predefined position during all ramps squeezing to $\beta^{*} \leq 2 \mathrm{~m}$, starting Oct. 26,01 . However, they were not used routinely for the ramps in the 2003 dai: 03 run.

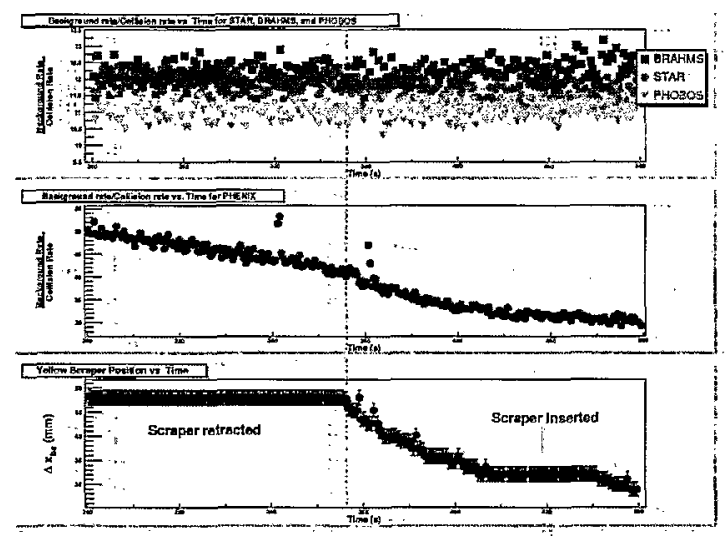

FIGURE , 3.: Background as a function. of scraper position during $A u$ operation. $\beta^{*}=2 m$ at all IRs.

The scrapers were also used-in an attempt to prevent experimental background during storage. Figure 3 shows the yellow scraper position and the experimental background rates based on ZDC [4] signals during fill 1759 . The yellow collimator was moved in by about $20 \mathrm{~mm}$ horizontally after orienting it such that it was parallel to the direction of the beam. There is no visible effect on the background signal except a small decrease in the
TABLE 2: List of tun - configurations during the rhic_dau_03 run. a $=\operatorname{IR} 6 \& 8, b=I R 2, c=I R 10$. "chg." refers to 'changing'; i.e. the cavity status changed rapidly during this period.

\begin{tabular}{|c|c|c|c|c|c|c|c|}
\hline \multirow[t]{2}{*}{ date } & \multirow[t]{2}{*}{ fill } & \multicolumn{3}{|c|}{$\beta^{*}(\mathrm{~m})$} & \multirow[t]{2}{*}{ n.o.b. } & \multicolumn{2}{|c|}{$\mathrm{RF}$. } \\
\hline & & $\mathrm{a}$ & $\mathrm{b}$ & c & & B & $Y$ \\
\hline bef: $01 / 11$ & - & 2 & 2 & 2 & $55 \because$ & n.a. & n.a. \\
\hline $01 / 11$ & 2715 & 2 & 2 & 2 & 110 . & n.a. & n.a. \\
\hline $01 / 17$ & 2758 & 2 & 2 & 2 & 110 . & on & on \\
\hline $01 / 21-23 \ldots$ & 2799 & 2 & 2 & 2 & 110 . & chg. & $\operatorname{chg}$. \\
\hline $01 / 24$ & 2812 & 2 & 2 & 2 & 110 & on & off \\
\hline 01/30: & 2843 . & 2 . & 2 & 2 & 110 & off & off. \\
\hline $02 / 02$ & 2883 & 2 & 2 & 2 & 110. & $\therefore$ on & on \\
\hline $02 / 09$. & 2952 & 2 & 2 & 4 & $110^{\circ}$ & on & on \\
\hline $02 / 24$ & 3056 & 2 & 2 & 4 & 110 . & off & on \\
\hline $02 / 27$ & 3070 & 2 & 2. & 4 & 55 & off & on \\
\hline $03 / 05$ & 3108 & 2 & 3 & 3 & 55 & off - & on \\
\hline $03 / 11$ & 3154 & 2 & 3 & 3 & $55:$ & on & on. \\
\hline $03 / 18$ & $3221^{\circ}$ & 2 & 3 & 3 & $55^{\cdots}$ & off & on \\
\hline $03 / 19 \therefore$ & 3235 & 2 & 3 & 3 & $55:$ & on & on: \\
\hline
\end{tabular}

PHENIX rates. Apparently, the scrapers had no significant effect on the experimental backgrounds at any time during the au_ 01 run. This leads to the conclusion that the background in the experiments was mainly due to local causes such as bearm-gas.interactions or colliding beams. Attempts to use the crystal for further experimental background reduction during the au_01 and dau_03 run were unsuccessful.

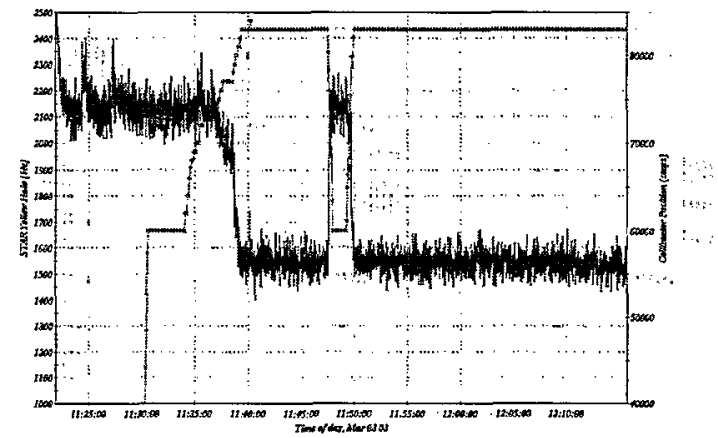

FIGURE 4. STAR ZDC background signal (line) and yellow. vertical collimator position (stars) during the dAu store 3094:

Table 2 gives an overview of the different running configurations during the dau_03 run. The usage of the storage cavities (RF B and Y) would mainly cause higher debunching rates, increasing the necessity of gap cleaning. The different $\beta^{*}$. values as well as the number of bunches (n.o.b.) likely lead to losses and background problems especially during the ramp development phase. In general; background conditions relaxed when $\beta^{*}$ was increased at IR10 and later at IR2. However, experimental background conditions did require collimation for the, most 
part of the run. Figure 4 shows a background signal from the STAR detector associated with background caused by the yellow (i.e. Au) beam: When the yellow vertical scraper is moved in (i.e. towards higher number of steps), the background signal drops by some $30 \%$ : Collimation clearly helped to reduce the background. Therefore effects local to the individual IR can be excluded as a sole cause for high background conditions. Collimators were used for background reduction more or less during every fill but the collimation efficiency did not appear to be sufficient.:

\section{POLARIZED PROTON OPERATION}

During both polarized proton runs beam was ramped to $\gamma \approx 107$. pp_02 used $\beta^{*}=3 \mathrm{~m}$ at all IRs while pp,03 used several settings. They are listed in table 3 below. In FY02 there was no need to use the scrapers during the ramp and neither scraper nor crystal were used routinely at any time during the pp_02 run. Figure 5 shows the effect of both, crystal and scraper, on experimental backgrounds in STAR (top) and BRAHMS (center). The experimental background rates are normalized to the collision rate. While the crystal has no obvious effect on the background signal in either IR, the retracted scraper increases the signal at IR6 (STAR) by $6 \%$ while decreasing it slightly at IR2 (BRAHMS). Background signals: were derived from the experimental luminosity : monitors (ZDC), which are situated close to the beam pipe. However, when looking at other signals from STAR [5]

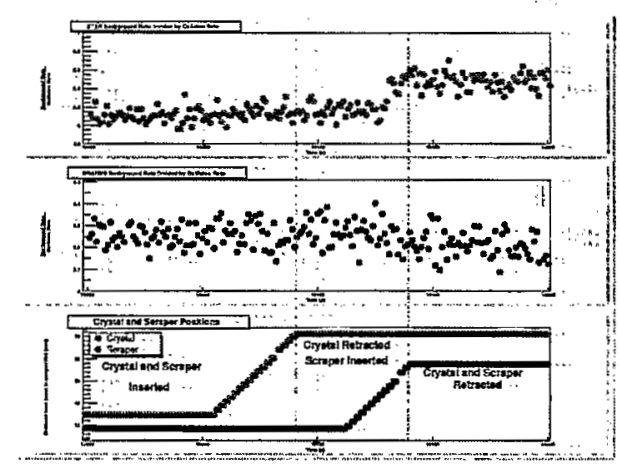

FIGURE 5. Background signals as a function of collimator position during fill 2185 in run pp_02.

coming from a detector some $2 \mathrm{~m}$ away from the beam pipe, background is increased when the scraper is moved.. in. The results from the crystal are inconclusive since.it increases as well as decreases the background compared to the scraper being in alone. In either case, the rates are higher than with both devices out by several $10 \%$. The crystal experiment was repeated in the following year in the dau. 03 run and is reported in [6]. It confirmed that a
TABLE 3. List of configurations during the pp.03 run with 55 bunches. "-" refers to "off" or "no collisions". A snake value of 1.88 refers to a full snake in one.ring plus $88 \%$ in the other, $a=\operatorname{IR} 6 \& 8, b=I R 2 \& 10,(*) I R 2$ only

\begin{tabular}{|c|c|c|c|c|c|c|c|c|}
\hline 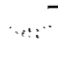 & \multirow[t]{2}{*}{ date : . } & \multirow[t]{2}{*}{ fill } & \multicolumn{2}{|c|}{$\beta^{*}(\mathrm{~m})$} & \multirow[t]{2}{*}{ snk. } & \multirow{2}{*}{$\mid \begin{array}{r}\text { rotrs. } \\
\text { (IR) }\end{array}$} & \multicolumn{2}{|c|}{$\mathrm{RF}$} \\
\hline & & & $\mathrm{a}$ & b & & & B & $\mathrm{Y}$ \\
\hline & $04 / 08$ & 3427 & 2 & 2 & full & - & - & - \\
\hline$\because$ & $04 / 12$ & 3459 & 1 & 3 & full & - & - & - \\
\hline & 04/14-15. & 3480 & 1 & 3 & full : & - & on & on \\
\hline & $04 / 16^{\circ}$ & 3496 & 1 & 3. & $\therefore$ & - & - & - \\
\hline & $04 / 19$ & 3502 & 1 & 3 & 1.88 & - & - & - \\
\hline & $04 / 25$ & 3541 & $: 1$ & 3 & 1.88 & 8 & - & - \\
\hline & $04 / 30 \cdots$ & 3591 & 1 & 3 & 1.88 & 8 & on & on \\
\hline & $05 / 15$. & 3720 & $\because 1$ & 3 & 1.88 & $8 \& 6$ & on & on. \\
\hline 28 & $05 / 18$ & 3735 & $\because 1$ & 3 & 1.88 & $8 \& 6$ & on & - \\
\hline & 05/19-21 & 3744 & $\therefore$ & $10^{*}$ & 1.88 & - & on & - \\
\hline & $05 / 21$ & 3757 & 1 & 3 & 1.88 & $8 \& 6$ & on & - \\
\hline & $05 / 23 \cdots$ & 3767 & 1 & - & 1.88 & $8 \& 6$ & on & on \\
\hline
\end{tabular}

collimation system including the crystal as primary col- . limator at its current location cannot reduce experimental backgrounds. During a special experiment in 2002 , several detector signals from PHENIX [7] were mon- $;$; itored during a dedicated end-of-fill background study. When the scrapers are moved in aggressively, the MUID (PHENIX: muon identification chambers, extending to meters away from the pipe) rate drops by a factor of 8 . while others, BBCLL1 and NTC. (both collision signals from detector components close to the beam pipe) remain. constant More details can be found in [2].

The FY03 polarized proton run again consisted : of many ramp commissioning periods. The various schemes. are listed in table 3 . The include changes in $\beta^{*}$, as well as various states of the snake magnets (necded to maintain transverse polarization during the RHIC ramp) and ro-: tator magnets around PHENIX and STAR (needed to Iocally change transverse polarization into longitudinal po-. larization). They sum up to a total of eight commissioning periods in eight weeks! In addition, the $\beta$-squeeze approach was changed. Instead of squeezing during the en-ergy ramp; a dedicated squeeze-ramp was performed at flattop energy. In short, the conditions during run pp_03 were quite different from pp_02. Collimation was essential during most parts of the run. Background conditions were bad enough to prevent experiments from turning on some of their detectors.

Figure 6 shows background signals from. PHENIX: during the period when beams collided in IR6 and IR8 only. The scintillators were installed on either side close to the Q3 magnets, some 40 meters upstream of the IR. It turned out that their signal represented the background conditions for the MUID detector fairly well, indicating that background was caused by beam scraping in the ' triplet area. The signals are shown in percent of the value before collimation starts. When the horizontal jaw is 

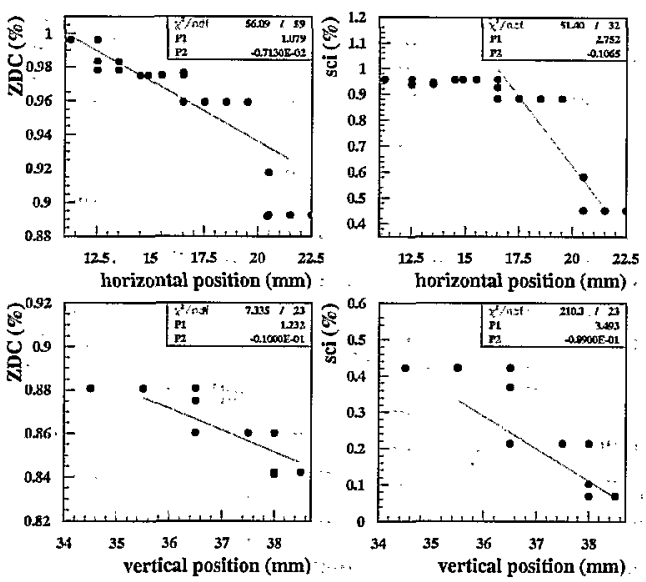

FIGURE 6. PHENIX scintillator and ZDC signals as a function of the collimator.jaw position during fill 3780 .

moved (top two figures), the slope of the linear fits differs by approximately a factor of 100 between the ZDC and scintillator signal. This difference is reduced to a factor 10 when the vertical jaw is moved closer to the beam. The horizontal jaw was moved first. Also, in this plane the drop of the scintillator signals begins later (by about $4 \mathrm{~mm}$ ) than the: decrease in the ZDC signal. However, while the the ZDC signal is reduced by about $16 \%$ only, the other signal drops to about $5 \%$ of its original value. At this point the MUID detectors could: be turned on. This difference is due to the low sensitivity of the ZDC detector to beam background in general.

\section{UPGRADE PLANS}

Anticipating higher beam currents; higher luminosity: and therefore higher backgrounds; the crystal will be removed and a conventional 2 stage system will be installed in its place [8]. The efficiency of the crystal collimation strongly depends on the local lattice function and was shown to have insufficient background removal efficiencies [6]. The 2 stage system includes two secondary horizontal collimators and one secondary vertical collimator: The primary collimator consists of both, a horizontal and vertical jaw. All collimators will be, for this upcoming run, positioned around PHENIX, in the warm straight section between the triplet (Q3) and Q4. Thus there will be three independently movable horizontal collimators and two vertical collimators per ring. The vertical secondary collimator will not be operational at the beginning of the next run. Fig. 7 shows a sketch of the collimator system as being installed. Each collimator will be furnished with four PIN diode loss monitors

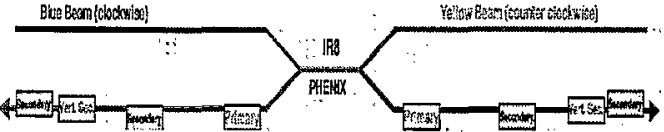

FIGURE 7. : Sketch of the planned conventional 2 stage collimation system.

allowing a feedback system based on the loss rate individual jaw.

In addition to the secondary collimators; stainless steel shielding [9] is being installed in the tunnel around the PHENIX IR. Thus, a significant reduction of the background rates is expected in particular. in the PHENIX Muon Identification chambers (MUID) for the next run.

\section{CONCLUSION}

Background conditions were particularly challenging during the past two runs; dau_03 and pp_03, most likely due to the horizontal crossing angles (dAu), the ramping and squeezing scheme (pp) and in general the high number of running configurations during both runs. Although background could be reduced at various locations with the primary collimators alone it remained difficult, if not impossible, to reduce background rates to a tolerable level at all experiments at the same time for the most part of the last two runs. The existing crystal collimator at its location could not enhance the overall collimation efficiency. Therefore, additional secondary collimators are being installed while the crystal collimator is dismounted. The new, mainly horizontal system, will be operational for the next run.

\section{REFERENCES}

1. R.P. Fliller II et. al: "The:Two Stage Crystal Collimator ..." for RHIC"; Proceedings of the PAC 01, Chicago (2001).....

2. A. Drees et al.,"RHIC Collimator Performance",Proceedings of the EPAC 02, Paris; June 2002 .

3. A. Drees, L. Ahrens et al. "Abort Gap Cleaning in RHIC", Proceedings of the EPAC 02, Paris, June 2002.

4. C. Adler, et al., nucl-ex/0008005.

5. W. Christie, "STAR", RHIC Retreat 2003, http://www.rhichome.bnl.gov/AP/RHIC2003/Retreat/.

6. R: Fliller et. al. "Crystal Collimation at RHIC", these Proceedings.

7. M. Perdekamp, "PHENIX", RHIC Retreat 2003, http://www.rhichome.bnl.gov/AP/RHIC2003/Retreat/.

8. R. Fliller, A. Drees; "Collimation", RHIC Retreat 2003, ; http://www.rhichome.bnl.gov/AP/RHIC2003/Retreat/

9. Kin :Yip, "Shielding",RHIC Retreat 2003, http://www.rhichome.bnl.gov/AP/RHIC2003/Retreat/. 